\title{
The Study of Tracking Technology for Mobile Robot Manipulator
}

\author{
Hailiang Yang \\ Zhonghuan Information College Tianjin University of Technology, Tianjin 300380, China. \\ yhailiang022@163.com.
}

Keywords: Mobile manipulator, Nonlinear uncertainty systems, Tracking control

\begin{abstract}
Mobile robot arm with the advantages of mobile platform and operating arm can have unlimited space and flexible operation. In recent years, the control of such systems has caused great attention in the field of domestic and foreign control. At the same time, the mobile manipulator is a typical nonlinear system with multi input, multi output, time-varying, strong coupling characteristics. It is difficult to use the traditional linear theory to study. Therefore, it has a great significance and good application prospects to study the tracking control of nonholonomic wheeled mobile manipulator.
\end{abstract}

\section{Introduction}

Mobile robots is an important branch of robotics. It is developing in the direction of the intelligent and diversification with the rapid development of sensor technology, computer science, artificial intelligence and other related subjects. It is becoming more and more widely, almost penetrated into all fields.

Mobile manipulator system is a mobile robot system by a mechanical arm fixed on a mobile robot platform. It must coordinate the movement of robot and mechanical arm with two functions of mobile and operation. The initial direction of the mobile manipulator mainly used in aerospace, now it is throughout the land and underwater environment, and have widely application prospect in industry, agriculture, medical, etc.

\section{Mathematical model of wheeled mobile robot}

Wheeled mobile manipulator system is composed of a mechanical arm and a wheeled mobile robot platform. The constraints of the wheeled mobile platform and the ground are nonholonomic constraints. The arm fixed on a mobile platform can be seen as a complete restraint. This paper mainly discusses the overall kinematic model and dynamic model about a simple arm of a wheeled mobile robot, which can provide reference for the subsequent tracking control.

\subsection{Kinematic model of wheeled mobile robot}

Assuming that the research of mobile robot is composed of rigid frame and rigid wheel and runing on horizontal plate, a kinematic model is established for Fig.1. XOY is the global Cartesian coordinate system where is the robot motion plane, $\mathrm{C}$ as the center of the robot. $\mathrm{X} 1$ is defined the direction of the moving car. $\theta$ is the mobile direction angle of the car. The coordinates of $\mathrm{C}$ point as the center of the car is $(\mathrm{x}, \mathrm{y})$. so using the three-dimensional vector $\xi=(\mathrm{x}, \mathrm{y}, \theta)^{\mathrm{T}}$ can completely describe the position of the mobile car.

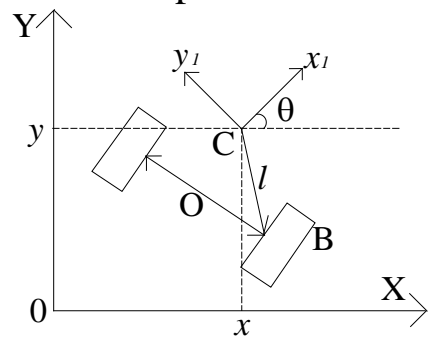

Fig.1 Kinematics model of wheeled mobile robot

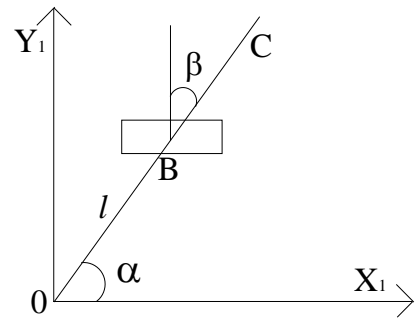

Fig.2 Structure chart of wheeled mobile robot

Fig. 2 is a block diagram of the mobile car fixed wheel. Point B is the center of the wheel. B are 
expressed as length $\mathrm{CB}=\mathrm{l}$ and angle $\alpha$ in the position of the coordinate system (x1, $\mathrm{y} 1)$. The direction of the wheel plane relative to the $\mathrm{CB}$ is represented by constant angle $\beta$. The wheel angle of rotating around its horizontal axis is $\varphi(t)$, the wheel radius is set to $\mathrm{R}$. The wheel state is given by four constants of $\alpha, \beta, \mathrm{l} 、 \mathrm{r}$. Motion state is determined by the time-varying angle $\varphi(t)$.

The kinematic model of the mobile robot is as follows:

$$
\left(\begin{array}{c}
\dot{x} \\
\dot{y} \\
\dot{\theta}
\end{array}\right)=\frac{i r}{2}\left(\begin{array}{cc}
\frac{\cos (\theta-\beta)}{\cos \beta} & \frac{\cos (\theta+\beta)}{\cos \beta} \\
\frac{\sin (\theta-\beta)}{\cos \beta} & \frac{\sin (\theta+\beta)}{\cos \beta} \\
-\frac{1}{l \cos \beta} & \frac{1}{l \cos \beta}
\end{array}\right)\left(\begin{array}{l}
\dot{\theta}_{l} \\
\dot{\theta}_{r}
\end{array}\right)
$$

\subsection{Kinematic model of wheeled mobile robot arm}

The mathematical model of the mobile robot and its two rotating joint is shown in Fig.3. The following are the various parameters to be considered when establishing the model.

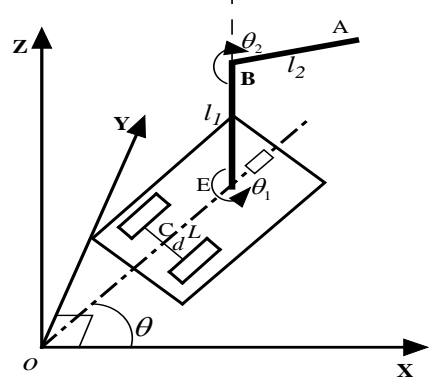

Fig.3 The model of mobile robot and its joint manipulator

The output of the mobile robot arm is connected with the position of the robot body, the left and right wheel and the angular velocity of the connecting rod. After deduction, the kinematic constraint of the mobile manipulator is as follows:

$$
\left[\begin{array}{c}
\dot{x}_{E} \\
\dot{y}_{E} \\
\dot{x}_{A} \\
\dot{y}_{A} \\
\dot{z}_{A}
\end{array}\right]=\left[\begin{array}{ccccc}
\cos \theta & -\sin \theta & 0 & 0 & 0 \\
\sin \theta & \cos \theta & 0 & 0 & 0 \\
0 & 0 & \cos \theta & -\sin \theta & 0 \\
0 & 0 & \sin \theta & \cos \theta & 0 \\
0 & 0 & 0 & 0 & 1
\end{array}\right]\left[\begin{array}{lllll}
\frac{r}{2} & \frac{r}{2} & 0 & 0 & 0 \\
-\frac{L \cdot r}{d} & \frac{L \cdot r}{d} & 0 & 0 & 0 \\
-\frac{r}{d} J_{11} & \frac{r}{d} J_{11} & J_{11} & J_{12} & J_{13} \\
-\frac{r}{d} J_{21} & \frac{r}{d} J_{21} & J_{21} & J_{22} & J_{23} \\
-\frac{r}{d} J_{31} & \frac{r}{d} J_{31} & J_{31} & J_{32} & J_{32}
\end{array}\right]\left[\begin{array}{c}
\dot{\theta}_{1} \\
\dot{\theta}_{r} \\
\dot{\theta}_{1} \\
\dot{\theta}_{2} \\
0
\end{array}\right]
$$

\section{Dynamic model of wheeled mobile manipulator}

The dynamic equations of the mobile manipulator can be obtained from the Lagrange equation of nonholonomic systems:

$$
\frac{d}{d t}\left(\frac{\partial L}{\partial \dot{q}}\right)-\frac{\partial L}{\partial q}=Q+A^{T}(q) \lambda
$$

Including: $\mathrm{L}$ is the Lagrange function; $\mathrm{T}$ is the kinetic energy of the system; $\mathrm{V}$ is the potential energy of the system; $\mathrm{q}$ is the generalized coordinates; $\mathrm{Q}$ is the forces acting on the generalized coordinates; $A^{T}(q)$ is the constraint matrix; $\lambda$ is the Lagrange multiplier vector;

$$
A(q) \dot{q}=0
$$

The dynamic model of a mobile robot manipulator is obtained by combining the nonholonomic constraint equations (5):

$$
M(q) \ddot{q}+N(q, \dot{q})=B(q) \tau+A^{T}(q) \lambda
$$




\section{Design of mobile manipulator controller}

A feedback controller is designed to control the input torque, so that the output of the mobile manipulator system is close to the desired path. Because of the nonlinear and coupling of the system, the feedback linearization method is used to realize the linearization of the system.

The state space of the mobile manipulator system is described as:

$$
\left\{\begin{array}{l}
\dot{q}=S(q) v \\
\dot{v}=\left(S^{T} M S\right)^{-1}\left(\tau-S^{T} M \dot{S} v-S^{T} N\right)
\end{array}\right.
$$

The vector $q, v$ is a minimal vector group which can describe the motion state of the mobile manipulator system. They can constitute the state vector of the system, so the state equation of the mobile manipulator system is as follows:

$$
\dot{x}=\left[\begin{array}{c}
S v \\
f_{1}
\end{array}\right]+\left[\begin{array}{c}
0 \\
\left(S^{T} M S\right)^{-1}
\end{array}\right] \tau
$$

Apply the following nonlinear feedback:

$$
\tau=\left(S^{T} M S\right)\left(u-f_{1}\right)
$$

Equation (8) can be reduced to the following form:

$$
\dot{x}=f(x)+g(x) u
$$

It can be seen that the simplified equation is a typical nonlinear system, so we can use the method of nonlinear feedback to achieve precise control for the system.

\section{Simulation study of tracking controller}

In the simulation experiment, the following parameters are used in the nonholonomic mobile manipulator: $m_{0}=20 \mathrm{~kg}, m_{1}=5 \mathrm{~kg}, m_{2}=5 \mathrm{~kg}, l_{1}=0.2 \mathrm{~m}, l_{2}=0.2 \mathrm{~m}, \mathrm{~L}=0.3 \mathrm{~m}, \quad \mathrm{r}=0.1 \mathrm{~m}$, $\mathrm{d}=0.2 \mathrm{~m}, \quad J_{0}=1.5 \mathrm{kgm} 2, \quad J_{1}=J_{2}=0.1 \mathrm{kgm} 2$, Initial position coordinates of mobile manipulator: $q=\left[\begin{array}{lllll}x_{E} & y_{E} & \theta & \theta_{1} & \theta_{2}\end{array}\right]^{T}=\left[\begin{array}{lllll}0 & 0 & 0 & \pi / 4 & \pi / 4\end{array}\right]$ 。 Below are the simulation results of the center of mass of the moving platform to the specified path.

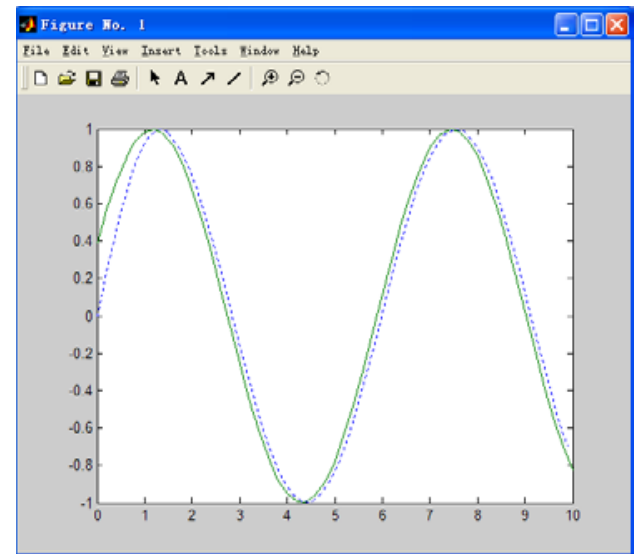

Fig. 4 Sine curve tracking simulation

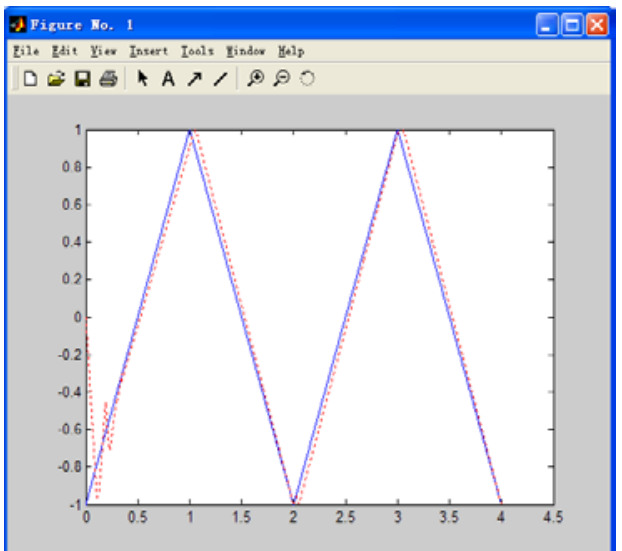

Fig. 5 Triangle-wave curve tracking simulation

From the simulation results, the mobile manipulator system controller for a given path that we have designed can have a good tracking effect. It can meet the needs of the actual control system.

\section{Summary}

In this paper, the application and design method of nonlinear feedback exact linearization method in nonlinear control system is introduced in detail. The mobile manipulator model was linearized and decoupled. The design of the controller for the output tracking of nonholonomic mobile manipulator is presented. The simulation results show that the control method can realize 
the output tracking of nonholonomic mobile manipulator, which can meet the needs of the actual control system.

\section{References}

[1] Jiang Lin, Yan Jihong, Zang Xizhe, Zhao Jie. Research on the motion control of mobile manipulator in all directions [J]. Journal of Xi'an Electronic and Science University, 2008, 35(4): 733-738

[2] V. Padois, J.-Y. Fourquet, P. Chiron. Kinematic and dynamic model-based control of wheeled mobile manipulators: a unified framework for reactive approaches. Robotica, Mar2007, Vol. 25 Issue 2, 157-173.

[3] Yan Chaoqin. Study on intelligent control of mobile manipulator (D). Xi'an Electronic and Science University, master's thesis, 2005.01

[4] Zhang Shuosheng, Yu Da. Robust tracking controller design for wheeled mobile robot. Journal of Beijing University of Science and Technology, 2003, 25(2): 22-28

[5] Glenn D. White, Rajankumar M. Bhatt, Venkat N. Krovi. Dynamic redundancy resolution in a nonholonomic wheeled mobile manipulator. Robotica, Mar2007, Vol. 25 Issue 2, 147-156 